effect $^{3}$. However, a recent work reported a similar scenario, in the absence of the Raman effect, involving the interaction of MMSs in multimode fibres ${ }^{10}$. In this latter study, the authors revealed a remarkable process in which, as a general rule, a transfer of power takes place from the higher-order modes towards the fundamental mode of the fibre.

It is interesting to note that such an irreversible transfer of power from higherorder modes towards the fundamental mode can have a purely thermodynamic origin, in the sense that it is thermodynamically advantageous for the optical beam to transfer its power into the fundamental mode of the system to increase the amount of entropy (disorder) in the system (see Fig. 1, bottom right, where $m_{x}$ and $m_{y}$ denote the mode numbers). This phenomenon is known as condensation of classical waves ${ }^{11}$.
It originates from the natural thermalization of the multimode beam towards a generalized Rayleigh-Jeans equilibrium distribution, whose divergence entails the macroscopic occupation of the fundamental mode of the fibre, whereas higher-order modes exhibit an energy equipartition. From a broader perspective, multimode fibres can be exploited to study a variety of phenomena in physics such as optical turbulence, whose possible regimes are enriched by the intricate mix of spatial and temporal effects. Besides multimode incoherent solitons, the spatiotemporal dynamics can be described by different formalisms inherited from weak Langmuir turbulence in plasma, or the long-range Vlasov formalism relevant to the description of gravitational effects, such as the dynamics of spontaneous formation and interaction of galaxies in the Universe ${ }^{11}$.
Antonio Picozzi and Guy Millot are at the Laboratoire Interdisciplinaire Carnot de Bourgogne, Université de Bourgogne, CNRS, Dijon, France. Stefan Wabnitz is at the Dipartimento di Ingegneria dell'Informazione, Università di Brescia, and INO-CNR, Brescia, Italy. e-mail: Antonio.Picozzi@u-bourgogne.fr;

Guy.Millo@u-bourgogne.fr; stefan.wabnitz@unibs.it

References

1. Dudley, J. M. \& Taylor, J. R. Supercontinuum Generation in Optical Fibers (Cambridge Univ. Press, 2010).

2. Russell, P. Science 299, 358-362 (2003).

3. Wright, L. G., Christodoulides, D. N. \& Wise, F. W. Nature Photon. 9, 306-310 (2015).

4. Richardson, D. J., Fini, J. M. \& Nelson, L. E. Nature Photon. 7, 354-362 (2013)

5. Agrawal, G. P. Nonlinear Fiber Optics 5th edn (Academic Press, 2013).

6. Majus, D. et al. Phys. Rev. Lett. 112, 193901 (2014).

7. Renninger, W. H. \& Wise, F. W. Nature Commun. 4, 1719 (2013).

8. Droques, M. et al. Opt. Lett. 37, 4832-4834 (2012).

9. Couairon, A. \& Mysyrowicz, A. Phys. Rep. 441, 47-189 (2007).

10. Buch, S. \& Agrawal, G. P. Opt. Lett. 40, 225-228 (2015).

11. Picozzi, A. et al. Phys. Rep. 542, 1-132 (2014).

\title{
DIFFRACTIVE OPTICS
}

\section{Tuning colour in flexible silicon}

Agile control of the perceived colour of objects is desired for many applications such as sensing and displays. Although it can be achieved by the interference from multiple layers of materials with different refractive indices, a solution that avoids the use of thick structures and different thicknesses for different colours, while having multiple colours tunable on the same flat surface, is highly sought after.

Now, Li Zhu and co-workers from the University of California Berkeley in the USA have experimentally demonstrated brilliant colours tunable from green to orange on a silicon thin film embedded in a flexible membrane (Optica 2, 255-258; 2015). The results are attractive for flexible optics applications.

Their approach was based on the design of high-contrast metastructures (HCMs), which are periodic structures made of a single-layer high-refractive-index material fully surrounded by low-index material, with a periodicity of nearly one wavelength. Their metastructures consist of an array of pixels embedded in a transparent, flexible polydimethylsiloxane membrane. Each pixel is composed of ribbons of silicon thin film, with a thickness of around one to two thousandths the thickness of a dollar bill. Unlike conventional approaches, the team used the same ribbon thickness and only changed the width of ribbon for different colours, making it easy to display a wide range of colours on the

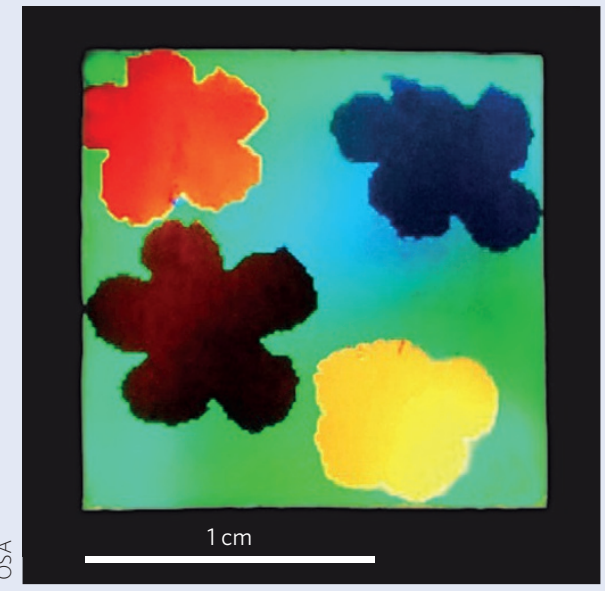

same flat surface. They also designed the period for the structure so that it is very close to the wavelength of visible light, and such that only two diffraction orders are allowed (the Oth and -1st) for both reflection and transmission.

The team explained that due to a large refractive index contrast between the ribbons and their surrounding medium, an interference effect is introduced on the optical wave reflected by the surface. The interference effect is very powerefficient due to the index contrast, leading to vivid colour determined by the spacing and width of the ribbons. Changing the spacing between the ribbons, by means of stretching the membrane, changes the wavelength at which strong constructive interference occurs, hence changing the colour of the surface.

"By carefully designing the structure dimension, it is possible for the interference of the eigenmodes to constructively enhance one particular diffraction order while destructively cancelling out the other orders. Because of the large refractive index contrast, this effect can be very broadband," said Connie Chang-Hasnain.

The researchers achieved $83 \%$ diffraction efficiency for the -1st reflection order. The colour of the sample can be tuned from green to orange - a $39 \mathrm{~nm}$ change in wavelength from 541 to $580 \mathrm{~nm}$ - by stretching the sample with only a $5 \%$ deformation, which corresponds to only a $25 \mathrm{~nm}$ change of the HCM period. They also demonstrated laser beam steering with more than 36 resolvable beam spots using the same effect, and they showed that the incident laser beam can be steered to more than a $5 \%$ deformation without beam width degradation.

"We will improve the design with two-dimensional structures to make it insensitive to diffraction polarization and to mitigate any bending issues in the structures. We also plan to use such mechanically sensitive material for bioimaging and bio-labelling applications," Chang-Hasnain told Nature Photonics. 